\title{
Panax notoginseng saponins elicits anti-cancer effect by modulating miR-760/SMAD4 signaling in osteosarcoma
}

\section{Type}

Research paper

\section{Keywords}

Apoptosis, osteosarcoma, SMAD4, Panax notoginseng saponins, miR-760

\begin{abstract}
Introduction

Osteosarcoma (OS) is one of the most common malignant bone tumors, with an incidence of 4-5 per million among children and teenagers. Panax notoginseng saponins (PNS) a derivatives from Panax notoginseng, are potent drugs that have many biological activities including antitumor effects.

However, there have been no reports focused on the effect of PNS on OS development.

Material and methods

MTT and flow cytometry was used to detect the proliferation and apoptosis of OS cells treated by PNS. The expression of miR-760 was identified by qPCR. Luciferase assay was performed to verify the target of miR-760. Western blot was used to detect the expression of target proteins. In vivo analysis was employed to confirm the antitumor effect of PNS.
\end{abstract}

\section{Results}

We tested the PNS effect on a large numbers of microRNAs (miRs) in OS cells, we found that PNS significantly reduced miR-760. Also, luciferase assay has shown SMAD4 to be the target gene of miR-760 in OS cells. Rescue experiments were carried out to verify the relation between SMAD4 and miR-760. we found that overexpression of miR-760 can reverse the effect of PNS. PNS proven to exerts its effect through miR-760. Moreover, SMAD4 can reverse the effect of miR-760, indicating that miR-760 targets SMAD4 in OS cells.

\section{Conclusions}

This study extends our understanding of the effect of PNS in OS cell. we revealed a novel signaling pathway involved in the PNS mode of action, miR-760/SMAD4, this new pathway might be feasible as a target for the treatment of OS. 
$4 \quad$ Running title:

$5 \quad$ PNS inhibits osteosarcoma

$7 \quad$ Abstract

8 Introduction Osteosarcoma (OS) is one of the most common malignant bone tumors, with an

9 incidence of 4-5 per million among children and teenagers. Panax notoginseng saponins (PNS) a

10 derivatives from Panax notoginseng, are potent drugs that have many biological activities including

11 antitumor effects. However, there have been no reports focused on the effect of PNS on OS

12 development. Materials and methods MTT and flow cytometry was used to detect the proliferation

13 and apoptosis of OS cells treated by PNS. The expression of miR-760 was identified by qPCR.

14 Luciferase assay was performed to verify the target of miR-760. Western blot was used to detect the

15 expression of target proteins. In vivo analysis was employed to confirm the antitumor effect of PNS.

16 Results We tested the PNS effect on a large numbers of microRNAs (miRs) in OS cells, we found that

17 PNS significantly reduced miR-760. Also, luciferase assay has shown SMAD4 to be the target gene of

18 miR-760 in OS cells. Rescue experiments were carried out to verify the relation between SMAD4 and

19 miR-760. we found that overexpression of miR-760 can reverse the effect of PNS. PNS proven to

20 exerts its effect through miR-760. Moreover, SMAD4 can reverse the effect of miR-760, indicating

21 that miR-760 targets SMAD4 in OS cells. Conclusions This study extends our understanding of the 
22 effect of PNS in OS cell. we revealed a novel signaling pathway involved in the PNS mode of action, miR-760/SMAD4, this new pathway might be feasible as a target for the treatment of OS.

25 Keywords: Panax notoginseng saponins, miR-760, SMAD4, osteosarcoma, apoptosis

\section{Introduction}

Osteosarcoma (OS) is one of the most common malignant bone tumors which accounts for approximately $60 \%$ of bone malignancies ${ }^{1,2}$. It has become the most frequent cause of cancer-related

30 death in children ${ }^{3}$. At present, the standard treatment for OS is surgery along with chemotherapy using doxorubicin and cisptatin ${ }^{4}$. Despite great advances, the efficacy of OS treatment remains unsatisfactory, high recurrence and drug resistance make the 5-year survival rate of OS less than $30-40 \% 5,6$. Therefore, understanding the molecular mechanisms underlying the initiation and recurrence of OS is highly essential for developing novel therapeutic methods.

Over the past few decades, numerous dietary and botanical natural compounds or synthesized compounds have been studied on there their properties that can prevent tumorigenesis ${ }^{7,8}$. Panax

notoginseng saponins (PNS), mainly derived from Panax notoginseng, are patent medicines that are

commonly used as a treatment for cardiovascular disorders, such as strokes, atherosclerosis, coronary 
pointed out that total PNS extracted from the caudexes and leaves have been used for improving

mental function, treating insomnia and alleviating anxiety. Other studies also demonstrated its

antioxidant, anti-inflammation, and anti-apoptosis effects ${ }^{12,13}$. For instance, $\mathrm{Hu}$ et al illustrated that

PNS may function as an extrinsic regulator by mitigating the oxygen-glucose

deprivation/reperfusion-induced cell injury in a dose-dependent manner. Zhou et al demonstrated that

PNS could attenuate cell inflammation by modulating CCL2-induced intracellular oxidative stress;

meanwhile, PNS pretreatment could inhibit apoptotic pathways by reducing Bax/Bcl-2 ratio and

Caspase 3,8,9 expressions. As in cancer therapy, PNS has been determined to attenuate lung cancer

growth through Met/miR-222 axis, and breast cancer metastasis via modulating miR-18a-regulated

Smad2 expression ${ }^{14}$. Meanwhile, another study reported that PNS treatment could inhibit cell

migration and invasion of in metastatic mouse breast cancer cell line ${ }^{15}$. Hence, PNS may function as a

potential tumor-suppressor in various cancers. However, the effect of PNS in the progression of OS

has not been studied.

MicroRNAs (miRNA/miR) are 20-25 nucleotides small non-coding RNAs which induce

degradation of target mRNAs or suppress translation of them through binding the targeting sequences

in the 3'-untranslated regions (UTR) of the target genes. Thus, microRNAs are considered to participate in the development of various tumors, including osteosarcoma, and regulate cell proliferation, apoptosis, and tumorigenesis through multiple signaling pathways ${ }^{16,17,18,19}$. 
Methods and materials

Osteosarcoma cell lines MG63 and HOS were obtained from the Chinese Academy of Science and were maintained in RPMI1640 (Gibco, NY, USA) medium containing 10\% FBS (Gibco, NY,

69 USA) with 100U/mL penicillin-streptomycin (Invitrogen, CA, USA). Cells were cultured in

70 RPMI1640 medium at $37^{\circ} \mathrm{C}$ in $5 \% \mathrm{CO}_{2}$. PNS was obtained from Sigma Chemical Co (St. Louis, MO,

71 USA). Pre-miR-760 oligonucleotide, pcDNA3.1/SMAD4 vector, and their negative control were

72 synthesized by Genepharma (Shanghai, China). $5 \times 10^{5}$ NSCLC cells were seeded into 6 well plates. 24

73 hours later, the oligonucleotides were transfected into MG63 and HOS cells (200 nM ) using

74 Lipofectamine 2000 (Invitrogen, CA, USA) according to the manufacturer's instructions. In the rescue

75 experiments exploring whether SMAD4 can reverse the effect of miR-760, OS cells were transfected

76 with $400 \mathrm{nM}$ of miR-760 mimic. Cell line stably expressing miR-760 was selected by G418

77 administration.

78

Cell proliferation measured by MTT assay

80 After transfection, $6 \times 10^{3}$ MG63 and HOS cells were seeded onto 96 well plates. MTT experiment was

81 performed to detect cell proliferation. After different concentrations of PNS treatment, 1\% MTT

82 (Beyotime, Shanghai, China) was added to the culture medium and incubated at $37^{\circ} \mathrm{C} .4 \mathrm{~h} \mathrm{later}$, the

83 supernatant was removed and DMSO was added into each well. After that, the OD value was detected with a microplate reader (Bio-Rad, USA) at $490 \mathrm{~nm}$. 
87 Total RNAs were extracted using TRIzol Reagent (Invitrogen, Carlsbad, CA, USA) according to the manufacturer's instruction. Total RNAs in the amount of $1 \mu \mathrm{g}$ were reversely transcribed to cDNA using an RNA PCR Kit (Takara Biotechnology, Japan) which was used as a PCR template. To detect gene expression, quantitative real-time PCR (qRT-PCR) was performed using an iCycler iQ System with the iQ SYBR Green SuperMix (BioRad, USA) according to the manufacturer's instructions.

92 Small endogenous nuclear U6 snRNA was used as the internal control for the normalization of miRNA and GAPDH for mRNAs. The relative gene expression levels were calculated using $\left(2^{-\Delta \Delta C t}\right)$

94 method.

The protein samples were extracted from the MG63 and HOS cells using a lysis buffer. The protein concentrations were measured by BCA reagent kit (Thermo Fisher, Shanghai, China). $40 \mu \mathrm{g}$ protein was separated in $10 \%$ sodium dodecyl sulfate-polyacrylamide gel electrophoresis (SDS-PAGE)

100 followed by transferring to the polyvinylidene fluoride (PVDF) membranes. The blots were then 101 incubated in the 5\% skimmed milk for $2 \mathrm{~h}$ at room temperature. Thereafter, they were incubated with 102 the primary antibodies overnight at $4^{\circ} \mathrm{C}$. After wash, the blots were incubated with horseradish 103 peroxidase-conjugated (HRP) labeled goat anti-rabbit secondary antibody at room temperature for $2 \mathrm{~h}$.

104 Finally, the blot was treated with an ECL plus reagent kit (Pierce, Rockford, IL, USA) and visualized 

internal control.

109 MG63 and HOS cells were washed with PBS followed by digesting with trypsin. Then, the cells were

110 resuspended and stained with AnnexinV FITC/propidium iodide following the manufacturer's

111 protocols. Cell apoptosis was assessed in a flow cytometry system (Verse, BD, USA).

113 Luciferase assay

114 The wild-type and mutant 3'UTR of SMAD4 were synthesized and subcloned into the pGL3 vector

115 (Promega, Shanghai, China). MG63 and HOS cells were co-transfected with miR-760 mimics or

116 mimic control and the pGL3 vector carrying wild-type or mutant SMAD4-3'UTR. Subsequently,

$1170.15 \mu \mathrm{g}$ PRL-TK was transfected to MG63 and HOS cells as the internal control. Finally, the 118 luciferase activity was evaluated $48 \mathrm{~h}$ after transfection.

121 Zhejiang University approved all animal studies conducted in the current study (No. 2017056285).

$12215 \mathrm{Balb} / \mathrm{c}$ nude male mice aged 4-6 weeks were randomly divided into three groups including the

123 control group, PNS treatment group, PNS along with the miR-760 transfection group and housed

124 within a constant temperature of $18-23^{\circ} \mathrm{C}$ and $60 \%$ humidity. Normal MG63 cells in the amount of

$1251 \times 10^{6}$ were subcutaneously injected into the flank region of the mice in the control and PNS group. 
126 And miR-760 stably expressed MG63 cells were injected into the mice in the PNS+miR-760 group in

127 the same way. PNS was dissolved in normal saline was administered orally to these mice once a day at

128 the dose of $50 \mathrm{mg} / \mathrm{kg}$ for 10 days before tumor inoculation until the end of the study. The tumors size

129 were measured every two days using a caliper. The mice were sacrificed at day 30 under anesthesia

130 and tumors were excised. Mice were sedated with $10 \% 60 \mathrm{mg} / \mathrm{kg}$ ketamine hydrochloride (Ketasol;

131 Richter Pharma, Weis, Austria) and 2\% 10 mg/kg xylazine (Rompun; Bayer Health Care, Whippany,

132 NJ, USA). A part of the issues was placed in $10 \%$ formalin for histological and the remaining was

133 frozen at $-80^{\circ} \mathrm{C}$. The animal experiments were approved by the Ethics Committee of Zhejiang

134 Hospital (No. 2017056285).

Immunohistochemistry

137 After mice were anesthetized, the brain was quickly removed and hippocampus was rapidly dissected

138 in $4 \%$ paraformaldehyde for $2 \mathrm{~h}$. Then, the formalin-fixed and paraffin-embedded tissues were cut into

$1395 \mu \mathrm{m}$ sections on a cryostat (Leica CM1510, Leica Miceosystems, Heidelberg, Germany). Then, the

140 sections were microwaved in $0.01 \mathrm{M}$ sodium citrate $(\mathrm{pH}$ 6.0) for antigen retrieval. Afterward, the

141 sections were blocked via incubation in PBS containing $10 \%$ goat serum for $2 \mathrm{~h}$ at room temperature.

142 Therefore, these sections were incubated with the primary anti-SMAD4 antibody (Abcam, England) at

143 room temperature overnight. The HRP-labelled secondary antibody was then added and the positive

144 staining cells were detected using diaminobenzidine (DAB) according to the manufacturer's protocol. 

as the means $\pm \mathrm{SD}$. A student's t-test was used to analyze the differences between the two groups. One-way ANOVA was used to analyze the difference between multiple groups. $P<0.05$ was considered as statistical significance.

\section{Results}

PNS repressed cell proliferation of MG63 and HOS

MTT assay was used to investigate the effects of PNS on the proliferation of MG63 and HOS cells. As shown in Figure 1A and B, after treatment for 24 hours with control or different concentrations of PNS, the proliferation of MG63 and HOS cells were reduced by $0.5,1,5$ and $10 \mu \mathrm{M}$ of PNS. Also, 12, 18, and 24 but not 6 hours of $5 \mu \mathrm{M}$ PNS treatment inhibited the proliferation of OS cells (Figure 1B). Furthermore, MG63 and HOS cells were treated with $0.5,1$, and $5 \mu \mathrm{M}$ PNS for over 3 days, respectively. $0.5,1$, and $5 \mu \mathrm{M}$ of PNS exerts a significant inhibitory effect on both MG63 and HOS cells on days 2 and 3 (Figure $1 \mathrm{C}, \mathrm{D}$ ).

164 Cell proliferation is always associated with apoptosis. Thus, we next evaluated the cell apoptosis of 165 MG63 and HOS cells treated with PNS using flow cytometry. PNS induced apoptosis of MG63 and 166 HOS cells at the concentration of 1 and $5 \mu \mathrm{M}$ (Figure 2A, B). We further detected the 
indicated that 1 and $5 \mu \mathrm{M}$ PNS notably elevated the level of bax, cyto-c, and cleaved caspase3, however, reduced the expression of bcl-2 (Figure 2C).

PNS down-regulated miR-760 expression in MG63 and HOS cells

Quantitative real-time PCR was used to evaluate the expression of miRNAs. First, we screened the expression of several miRs in MG63 and HOS cells after PNS treatment. These miRs have been proved to be involved in the progression of OS. We found that PNS significantly down-regulated miR-128 and miR-760 expression at the dosage of $1 \mu \mathrm{M}$ (Figure 3A). Then, we selected miR-760 for further research. The results indicated that 1 and $5 \mu \mathrm{M}$ PNS notably inhibited the miR-760 expression (Figure 3B). Moreover, we found that 6 hours treatment of $1 \mu \mathrm{M}$ PNS didn't change the expression level of miR-760 while 12, 18, and 24 hours of treatment of $1 \mu \mathrm{M}$ PNS inhibited that (Figure 3C).

MiR-760 reversed the effect of PNS on MG63 and HOS cells

To confirm whether PNS exerts the anti-tumor effect through targeting miR-760, we carried out a rescue experiment. MTT and flow cytometry was used to evaluate the cell proliferation and apoptosis of MG63 and HOS cells. As suggested by the findings, PNS decreased the expression of miR-760. miR-760 transfection notably increased the expression of miR-760 compared to the PNS+miR-nc group (Figure 4A). Also, PNS treatment along with miR-760 transfection increased cell proliferation (Figure 4B) and reduced cell apoptosis compared to the PNS+miR-nc group (Figure 4C). 
189 To investigate the mechanism through which miR-760 reversed the effect of PNS, potential target

190 genes of miR-760 were predicted by bioinformatic algorithms targetscan7.2 and SMAD4 was selected

191 (Figure 5A). To further confirm whether miR-760 directly targets SMAD4 and suppresses its

192 expression, a firefly luciferase reporter was constructed containing a wild type or mutated type

193 fragment of the 3'-UTR of SMAD4 mRNA. The wild type or mutated luciferase reporters were

194 co-transfected into MG63 and HOS cells with miR-760 or miR-nc. The data showed that the

195 co-expression of miR-760 with wild type 3'UTR but not with mutant 3'UTR significantly inhibited

196 the luciferase activity (Figure 5B). Western blot was carried out to verify the prediction, the protein

197 expression of SMAD4 was notably inhibited by miR-760 overexpression and was promoted by

198 miR-760 knockdown (Figure 5C, D).

SMAD4 reversed the effect of $m i R-760$

201 As miR-760 targeted SMAD4 directly, we next investigated whether SMAD4 was involved in the

202 effect of miR-760 on cell proliferation or apoptosis. We found that PNS significantly up-regulated

203 SMAD4 expression at the dosage of 1 and $5 \mu \mathrm{M}$ (Figure 6A). miR-760 reversed the effect of PNS on

204 cell proliferation and apoptosis, while SMAD4 overexpression reversed this effect of miR-760 (Figure

$2056 \mathrm{~B}, \mathrm{C})$. Flow cytometry results showed miR-760 could decrease the apoptotic rates of MG63 and HOS

206 cells compared to the PNS+miR-760 group, while SMAD4 overexpression restored it (Figure 6D).

207 The western blot assay results revealed that miR-760 significantly inhibited the expression of

208 pro-apoptosis such as cyto-C, bax, and cleaved-caspase3, meantime promoted that of anti-apoptosis

209 protein such as bcl-2 compare to PNS group. Again, SMAD4 overexpression significantly increased 
210 the level of cyto-C, bax, and cleaved-caspase 3 while reduced that of bcl-2 compare to the

211 PNS+miR-760 group (Figure 6E).

212

213 PNS inhibited the OS tumor growth which could be reversed by miR-760

214 As Figure 7 A and B showed, PNS treatment significantly inhibited the tumor growth of MG63 cells

215 in mice. However, the pre-miR-760 transfected OS cell exerts a faster growth compared to the PNS

216 group. Moreover, PNS reduced the tumor weight while miR-760 reversed this effect of PNS (Figure

217 7C). To verify whether PNS and miR-760 modulate the expression of SMAD4 in vivo, we detected

218 the expression of SMAD4 in the tumor tissue. As expected, PNS notably promoted the expression of

219 SMAD4 while miR-760 reversed this elevation (Figure 7D).

221 Discussion

222 In the present study, we evaluated the anti-tumor effect of PNS on OS cells. First, we demonstrated

223 that PNS inhibit cell proliferation and promoted apoptosis in MG63 and HOS cells in vitro and in vivo.

224 Next, we investigated PNS mode of action in OS cells. Previous studies have shown that PNS exerts

225 vast and complex biological effects. For instance, PNS modulates the HIF- $1 \alpha /$ mitochondria/ROS

226 pathway in cisplatin-induced mitochondrial injury ${ }^{20}$. Also, PNS alleviates skeletal muscle insulin

227 resistance via regulating GLUT4 expression and IRS1-PI3K-AKT signaling ${ }^{21}$. Moreover, Panax

228 notoginsenoside saponins Rb1 regulates the expressions of Akt/ mTOR/PTEN signals in the

hippocampus after focal cerebral ischemia in rats ${ }^{22}$. 
MiRs participate in the regulation of various cell physiological processes such as proliferation,

apoptosis, and tumorigenesis for its critical inhibitory effect on their target genes. For instance, For

instance, Zhao et al proposed that miR-345-5p could suppress tumorigenesis of papillary thyroid

carcinoma by targeting SETD $7^{23}$. Previous studies have determined the interaction between PNS and

miRs. PNS inhibit lung cancer growth via modulating miR-222 $2^{24}$. It also promotes miR-181b

expression and the downstream mTOR/Akt/caspase-3 pathway to alleviate severe acute pancreatitis ${ }^{25}$.

236 Also, by targeting miR-155, PNS inhibited oxygen-glucose deprivation/reoxygenation-induced injury

237 in human SH-SY5Y cells ${ }^{26}$. The mechanism of PNS on miR in the OS has not been reported yet. We

238 first determined that PNS inhibits the expression of miR-760 in OS cells. Ectopic expression of

239 miR-760 can reverse the effect of OS which further proved their interaction.

240 MicroRNAs inhibit the translation of mRNAs or leads to its degradation by targeting the mRNAs.

241 Bioinformatics analysis and luciferase assay confirmed that miR-760 targeted SMAD4 in both MG63

242 and HOS cells. SMAD4 was initially known as "deleted in pancreatic carcinoma locus 4 (DPC4)"

243 which was identified as a candidate tumor suppressor gene $27,28,29$. It is a pivotal transducer of the

244 TGF- $\beta$ pathway and participates in the complex pathological process during tumorigenesis ${ }^{30,31}$.

245 Increasing evidence indicates that abnormal Smad4 expression is closely associated with different

246 human cancers including $\mathrm{OS}^{32,33}$. We assessed the expression of SMAD4 after the treatment of

247 different concentrations of PNS and revealed that PNS promotes the expression of SMAD4 in OS

248 cells. Next, we performed rescue experiments and found that SMAD4 overexpression reversed the

249 effect of miR-760 on cell proliferation and apoptosis. 
Apoptosis is a kind of programmed cell death that plays a crucial role in cancer development ${ }^{34}$.

251 Pro-apoptotic Bcl-2 family proteins such as Bax or Bak promotes the release of mitochondrial 252 cytochrome-C into the cytoplasm to activate caspase-9 then activates caspase-3, finally induce 253 apoptosis ${ }^{35,36}$. For instance, caspase- 3 was found to be related to cell apoptosis in mesangial cell and 254 ischemia/reperfusion injury progress ${ }^{37,38}$. Also, Zhang et al found that Bcl-2 and Bax expressions were expression and accordingly elevate the SMAD4 level. The aberrant expression of miR-760 and reduced anti-apoptotic protein bcl-2 level, finally initiate apoptosis.

sponged by long non-coding RNA ASB16-AS1 to functions as a tumor suppressor. Sometimes, the contradictive results will be found due to the difference in the experimental methods or regents. We will confirm it in our future studies. use of PNS in cancer therapy.

In conclusion, we firstly demonstrated that PNS inhibited cell proliferation and induced apoptosis of MG63 and HOS cells by regulating the expression of miR-760. Moreover, miR-760 regulated the apoptotic protein expression by directly targeting SMAD4. These findings extended our understanding of the effect of PNS in cancer progression. However, more work should be carried out for the clinical

\section{Declaration of absence of conflicts of interest}

No conflicts of interest exist in the present study. 


\section{References}

273 1. Heymann D. Metastatic osteosarcoma challenged by regorafenib. Lancet Oncol 2019;20:12-14.

274 2. Gambera S, Abarrategi A, Gonzalez-Camacho F, Morales-Molina A, Roma J, Alfranca A, et al.

275 Clonal dynamics in osteosarcoma defined by RGB marking. Nat Commun 2018;9:3994.

276 3. Xie L, Yao Z, Zhang Y, Li D, Hu F, Liao Y, et al. Deep RNA sequencing reveals the dynamic metastasis. Cell Death Dis 2018;9:772.

4. Wang Z, Liu Z, Wu S. Long non-coding RNA CTA sensitizes osteosarcoma cells to doxorubicin through inhibition of autophagy. Oncotarget 2017;8:31465-77.

5. Xie L, Yao Z, Zhang Y, Li D, Hu F, Liao Y, et al. Deep RNA sequencing reveals the dynamic metastasis. Cell Death Dis 2018;9:772.

6. Wang W, Li X, Meng FB, Wang ZX, Zhao RT, Yang CY. Effects of the Long Non-Coding RNA

7. Wang P, Cui J, Du X, Yang Q, Jia C, Xiong M, et al. Panax notoginseng saponins (PNS) inhibits breast cancer metastasis. J Ethnopharmacol 2014;154:663-71. 
9. Su P, Wang L, Du SJ, Xin WF, Zhang WS. [Advance in studies of Panax notoginseng saponins on pharmacological mechanism of nervous system disease]. Zhongguo Zhong Yao Za Zhi 2014;39:4516-21.

10. Zhang H, Li Z, Zhou Z, Yang H, Zhong Z, Lou C. Antidepressant-like effects of ginsenosides: A comparison of ginsenoside $\mathrm{Rb} 3$ and its four deglycosylated derivatives, $\mathrm{Rg} 3, \mathrm{Rh} 2$, compound $\mathrm{K}$, and 20(S)-protopanaxadiol in mice models of despair. Pharmacol Biochem Behav 2016;140:17-26.

11. Xiang H, Liu Y, Zhang B, Huang J, Li Y, Yang B, et al. The antidepressant effects and mechanism of action of total saponins from the caudexes and leaves of Panax notoginseng in animal models of depression. Phytomedicine 2011;18:731-38.

12. Hu SN, Wu YL, Zhao B, Hu HY, Zhu BC, Sun ZX, et al. Panax notoginseng Saponins protect cerebral microvascular endothelial cells against oxygen-glucose deprivation/reperfusion-induced barrier dysfunction via activation of PI3K/AKT/Nrf2 antioxidant signaling pathway. Molecules 2018;23:2781.

13. Zhou YJ, Chen JM, Sapkota K, Long JY, Liao YJ, Jiang JJ, et al. Pananx notoginseng saponins attenuate CCL2-induced cognitive deficits in rats via anti-inflammation and anti-apoptosis effects that involve suppressing over-activation of NMDA receptors. Biomed Pharmacother 2020;127:110139.

14. Wang P, Du X, Xiong M, Cui J, Yang Q, Wang W, et al. Ginsenoside Rd attenuates breast cancer metastasis implicating derepressing microRNA-18a-regulated Smad2 expression. Sci Rep 2016;6:33709.

15. Wang PW, Cui JG, Du XY, Yang QB, Jia CL, Xiong MQ, et al. Panax notoginseng saponins (PNS) 
inhibits breast cancer metastasis. J Ethnopharmacol 2014;154:663-671.

314 16. Zhang Z, Pi J, Zou D, Wang X, Xu J, Yu S, et al. microRNA arm-imbalance in part from

315 complementary targets mediated decay promotes gastric cancer progression. Nat Commun

$316 \quad 2019 ; 10: 4397$.

317 17. O'Connell RM, Zhao JL, Rao DS. MicroRNA function in myeloid biology. Blood $318 \quad 2011 ; 118: 2960-69$.

319 18. Girard M, Jacquemin E, Munnich A, Lyonnet S, Henrion-Caude A. miR-122, a paradigm for the 320 role of microRNAs in the liver. J Hepatol 2008;48:648-56.

321 19. Cano A, Nieto MA. Non-coding RNAs take centre stage in epithelial-to-mesenchymal transition. Trends Cell Biol 2008;18:357-59.

20. Li Q, Liang X, Yang Y, Zeng X, Zhong X, Huang C. Panax notoginseng saponins ameliorate Open Bio 2019. expression. FEBS Open Bio 2019;9:1008-19.

22. Yan YT, Li SD, Li C, Xiong YX, Lu XH, Zhou XF, et al. Panax notoginsenoside saponins Rb1

23. Zhao M, Wang KJ, Shang JB, Liang Z, Zheng WH, Gu JL. miR-345-5p inhibits tumorigenesis of papillary thyroid carcinoma by targeting SETD7. Arch Med Sci 2019;16:888-897. 
24. Yang Q, Wang P, Cui J, Wang W, Chen Y, Zhang T. Panax notoginseng saponins attenuate lung cancer growth in part through modulating the level of Met/miR-222 axis. J Ethnopharmacol 2016;193:255-65.

25. Liu MW, Wei R, Su MX, Li H, Fang TW, Zhang W. Effects of Panax notoginseng saponins on severe acute pancreatitis through the regulation of mTOR/Akt and caspase-3 signaling pathway by upregulating miR-181b expression in rats. BMC Complement Altern Med 2018;18:51.

26. Meng L, Lin J, Huang Q, Liang P, Huang J, Jian C, et al. Panax notoginseng Saponins Attenuate Oxygen-Glucose Deprivation/Reoxygenation-Induced Injury in Human SH-SY5Y Cells by Regulating the Expression of Inflammatory Factors through miR-155. Biol Pharm Bull 2019;42:462-67.

27. Yang G, Yang X. Smad4-mediated TGF-beta signaling in tumorigenesis. Int J Biol Sci 2010;6:1-08.

28. Miyaki M, Kuroki T. Role of Smad4 (DPC4) inactivation in human cancer. Biochem Biophys Res Commun 2003;306:799-804.

29. Holloway S, Davis M, Jaber R, Fleming J. A clinically relevant model of human pancreatic adenocarcinoma identifies patterns of metastasis associated with alterations of the TGF-beta/Smad4 signaling pathway. Int J Gastrointest Cancer 2003;33:61-69.

30. Schwarte-Waldhoff I, Schmiegel W. Smad4 transcriptional pathways and angiogenesis. Int J Gastrointest Cancer 2002;31:47-59.

31. Vizek M, Smejkal V, Palecek F. Fixed breathing frequency decreases end-tidal PCO2 in humans. Physiol Res 1991;40:257-60. 
32. Li Q, Pan X, Wang X, Jiao X, Zheng J, Li Z, et al. Long noncoding RNA MALAT1 promotes cell proliferation through suppressing miR-205 and promoting SMAD4 expression in osteosarcoma. Oncotarget 2017;8:106648-60.

33. Ma J, Huang K, Ma Y, Zhou M, Fan S. The TAZ-miR-224-SMAD4 axis promotes tumorigenesis in osteosarcoma. Cell Death Dis 2017;8:e2539.

34. Tompkins KD, Thorburn A. Regulation of Apoptosis by Autophagy to Enhance Cancer Therapy. Yale J Biol Med 2019;92:707-18.

35. Wang J. Cell Death Response to DNA Damage. Yale J Biol Med 2019;92:771-79.

36. Lee Y, Overholtzer M. After-Death Functions of Cell Death. Yale J Biol Med 2019;92:687-94.

37. Ying CJ, Wang SS, Lu Y, Chen L, Mao YZ, Ling HW, et al. Glucose fluctuation increased mesangial cell apoptosis related to AKT signal pathway. Arch Med Sci 2019;15:730-737.

38. Eken MK, Ersoy GS, Kaygusuz EI, Devranoglu B, Takir M, Cilingir OT, et al. Etanercept protects ovarian reverse against ischemia/reperfusion injury in a rat model. Arch Med Sci 2019;15:1104-1112.

39. Zhang Y, Zhang R, Ni HJ. Eriodictyol exerts potent anticancer activity against A549 human lung cancer cell line by inducing mitochondrial-mediated apoptosis, G2/M cell cycle arrest and inhibition of m-TOR/PI3K/AKT signaling pathway. Arch Med Sci 2019;16:446-452.

\section{Figure legends}

Figure 1. PNS inhibited the cell proliferation of MG63 and HOS cells at the dose-dependent or time-dependent manner. (A) MTT was used to evaluate the cell proliferation of MG63 and HOS cells after different concentrations of PNS treatment for $24 \mathrm{~h}$. (B) MTT was used to evaluate the cell 
proliferation of MG63 and HOS cells after $5 \mu \mathrm{M}$ PNS treatment at the indicated time. (C) The growth

curve of MEG63 cell line was made under PNS treatment for a period of 3 days. (D) The growth curve

of HOS cell line was made under PNS treatment for a period of 3 days. The results were presented as

the mean $\pm \mathrm{SD}, \mathrm{n}=6,{ }^{*} \mathrm{P}<0.05$ vs normal saline (NS).

Figure 2. PNS induced apoptosis of MG63 and HOS cells. (A) Flow cytometry was carried out to

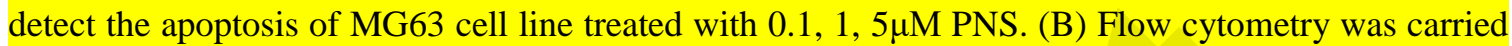

out to detect the apoptosis of HOS cell line treated with $0.1,1,5 \mu \mathrm{M}$ PNS. (C) Western blot was used

to investigate the apoptosis-related protein expressions in MG63 and HOS cells. The results were

presented as the mean $\pm \mathrm{SD}, \mathrm{n}=3,{ }^{*} \mathrm{P}<0.05$ vs NS.

results were presented as the mean $\pm \mathrm{SD}, \mathrm{n}=6,{ }^{*} \mathrm{P}<0.05$ vs NS.

Figure 4. MiR-760 overexpression reversed the effect of PNS. (A) qPCR was used to detect the expression level of miR-760 after PNS treatment or miR-760 transfection. (B) MTT was used to investigate the cell proliferation of MG63 and HOS cells after PNS treatment or the transfection of 
PNS treatment or the transfection of miR-760. The results were presented as the mean $\pm \mathrm{SD}, \mathrm{n}=6$,

${ }^{*} \mathrm{P}<0.05$ vs control, ${ }^{\#} \mathrm{P}<0.05$ vs $\mathrm{PNS}+\mathrm{miR}-\mathrm{nc}$.

Figure 5. SMAD4 is a direct target of miR-760. (A) Schematic representation of the miR-760

targeting sequences within the 3'-UTR of SMAD4. (B) Luciferase reporter assay was conducted in

MG63 and HOS cells following transfection with miR-760 or miR-nc, and together with Wt or Mut

SMAD4 3'UTR luciferase reporter plasmid. (C, D) The protein expression of SMAD4 in MG63 and

HOS cells transfected with miR-760, miR-nc, miR-760 inhibitor, or inhibitor control was detected by

western blotting. The results were presented as the mean $\pm \mathrm{SD}, \mathrm{n}=6,{ }^{*} \mathrm{P}<0.05$ vs miR-nc, ${ }^{\#} \mathrm{P}<0.05$ vs inhibitor control.

Figure 6. SMAD4 overexpression reversed the effect of miR-760. OS cells were transfected with 200nM miR-760 mimic and the SMAD4 overexpressing vectors. (A) Western blot was used to detect the expression level of SMAD4 after the treatment of PNS and the quantified results of western blot assay was presented. (B, C) MTT was used to investigate the cell proliferation of MG63 and HOS

412 cells after PNS treatment or overexpression of SMAD4. (D) Flow cytometry was used to investigate

413 the cell apoptosis of MG63 and HOS cells after PNS treatment or overexpression of SMAD4. (E)

414 Western blot was used to evaluate the apoptotic protein expressions of MG63 and HOS cells after PNS

415 treatment or overexpression of SMAD4. The results were presented as the mean $\pm \mathrm{SD}, \mathrm{n}=6, * \mathrm{P}<0.05$

416 vs PNS+miR-nc, ${ }^{\#} \mathrm{P}<0.05$ vs $\mathrm{PNS}+\mathrm{miR}-760$. 
419 The mice in each group were sacrificed and the tumors were excised and pictured. (B) Tumor sizes of

420 the mice in each group were measured and the growth curve was shown. (C) Tumor weight was

421 measured in each group. (D) IHC was performed to evaluate the expression of SMAD4 in the tumor

422 tissues. The results were presented as the mean $\pm \mathrm{SD}, \mathrm{n}=3,{ }^{*} \mathrm{P}<0.05$ vs control, ${ }^{\#} \mathrm{P}<0.05$ vs $\mathrm{PNS}$. 
A

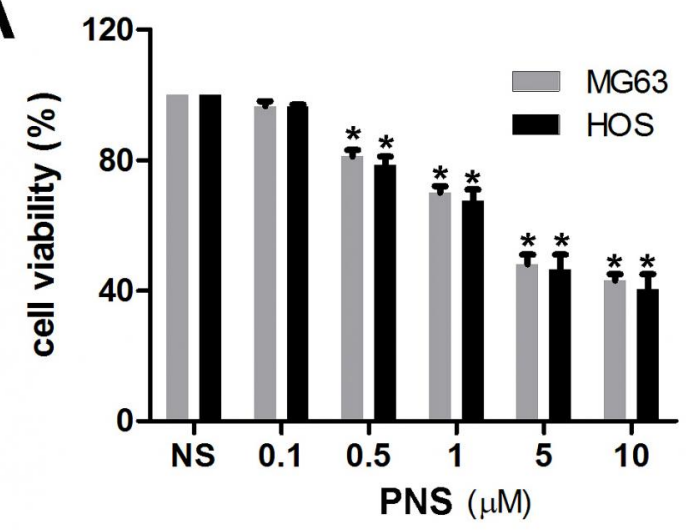

MEG63

C

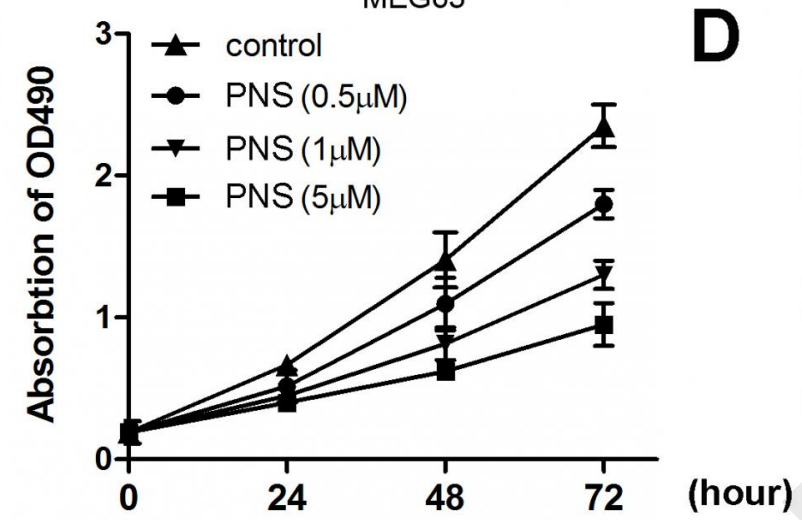

B

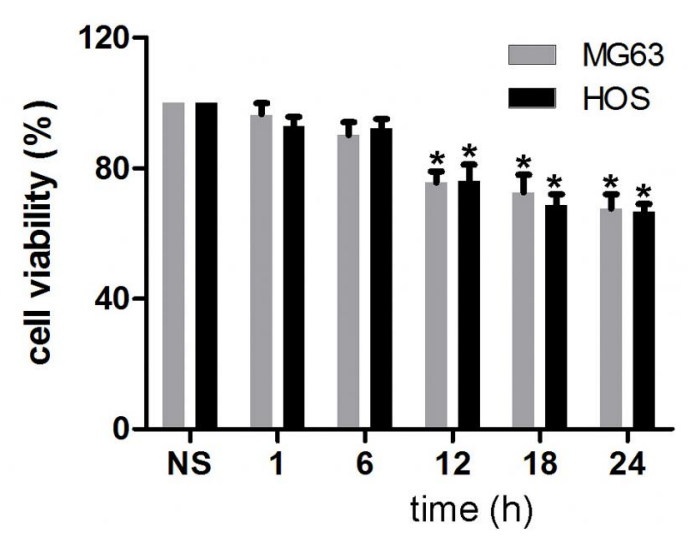

HOS

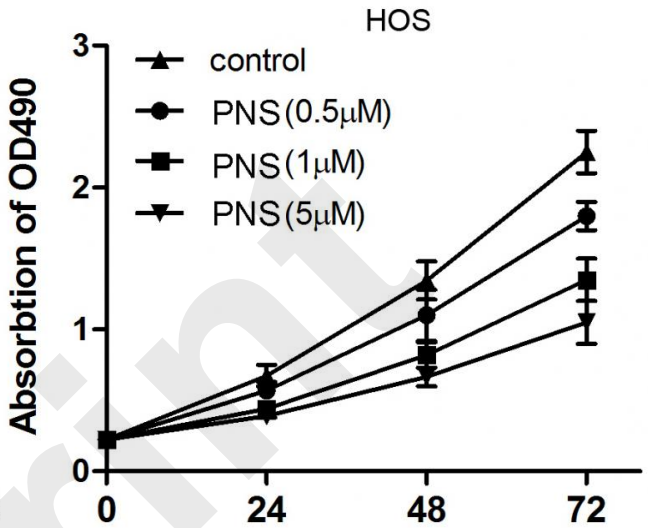


A
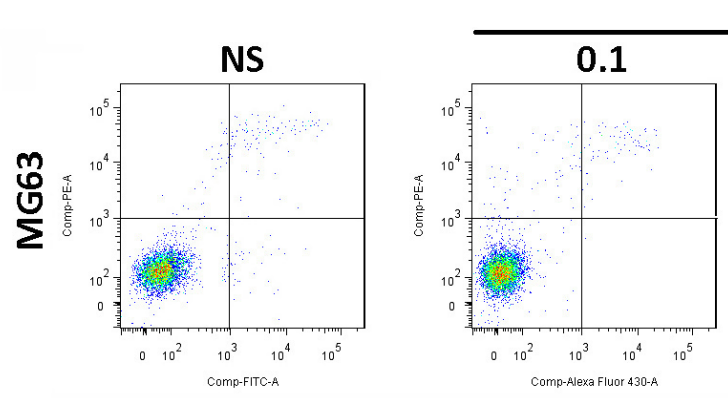

PNS $(\mu \mathrm{M})$
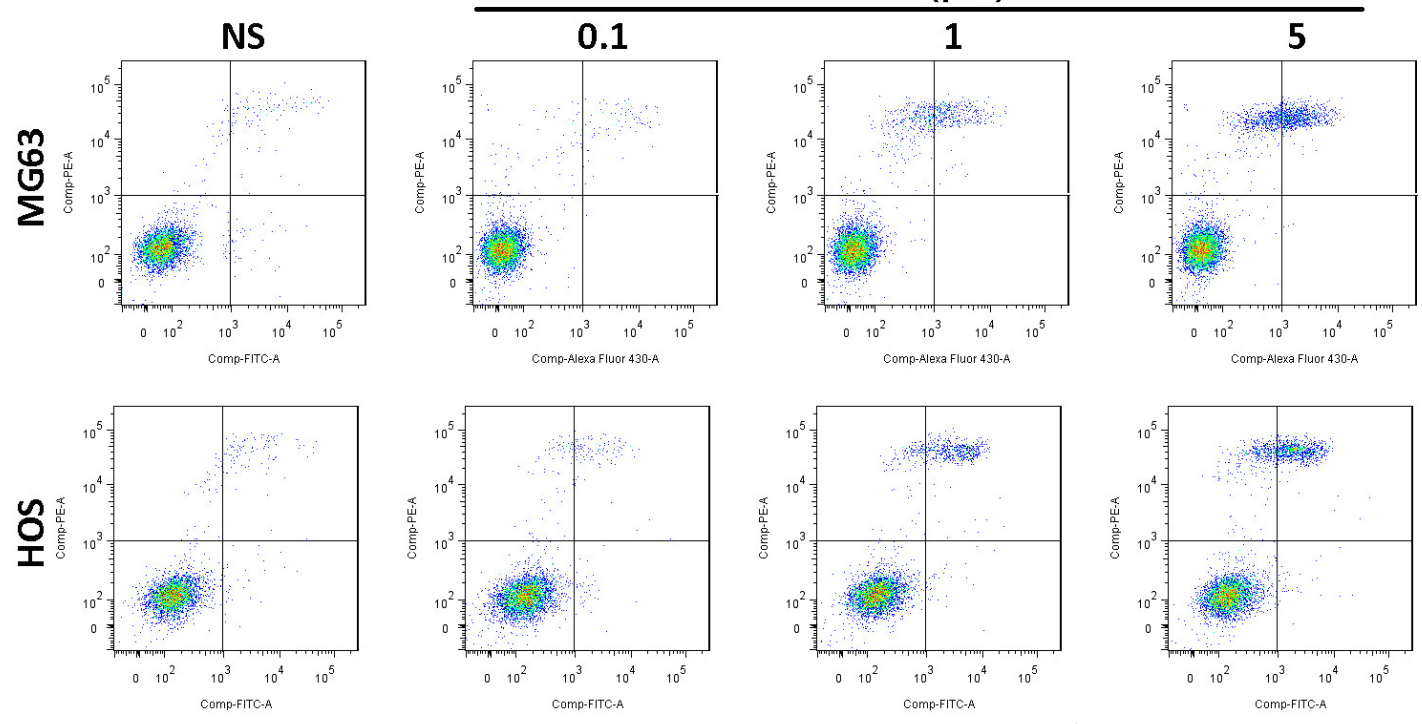

B

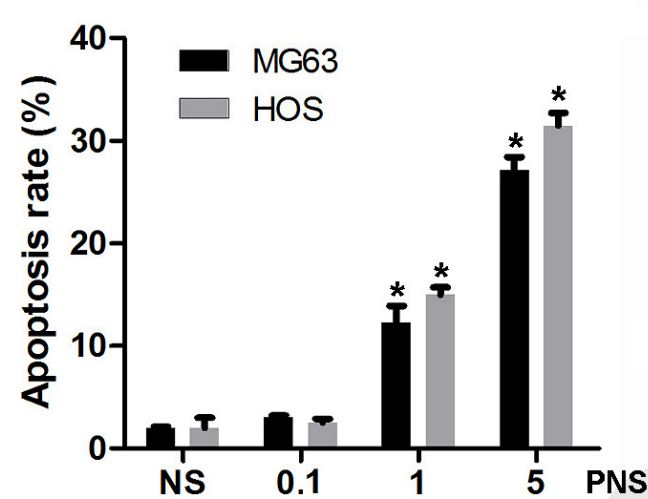

C
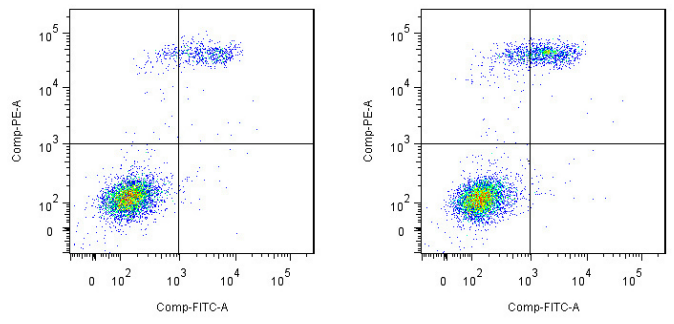

C

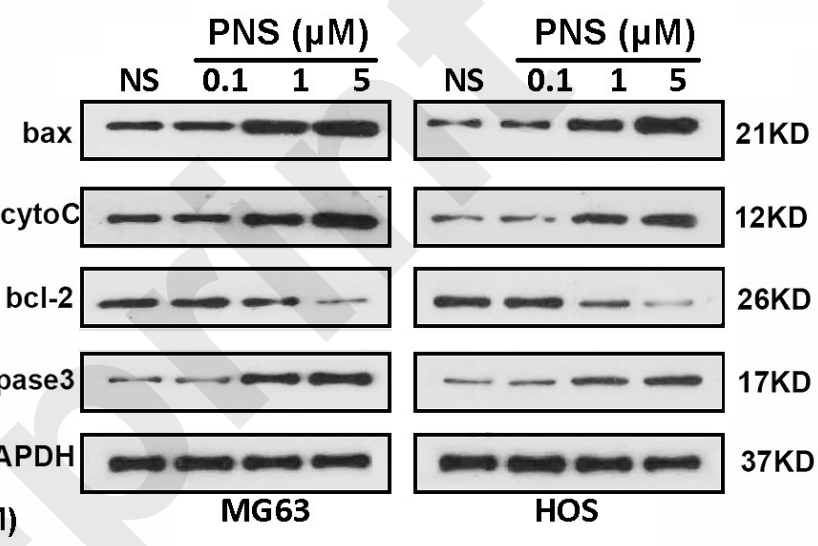



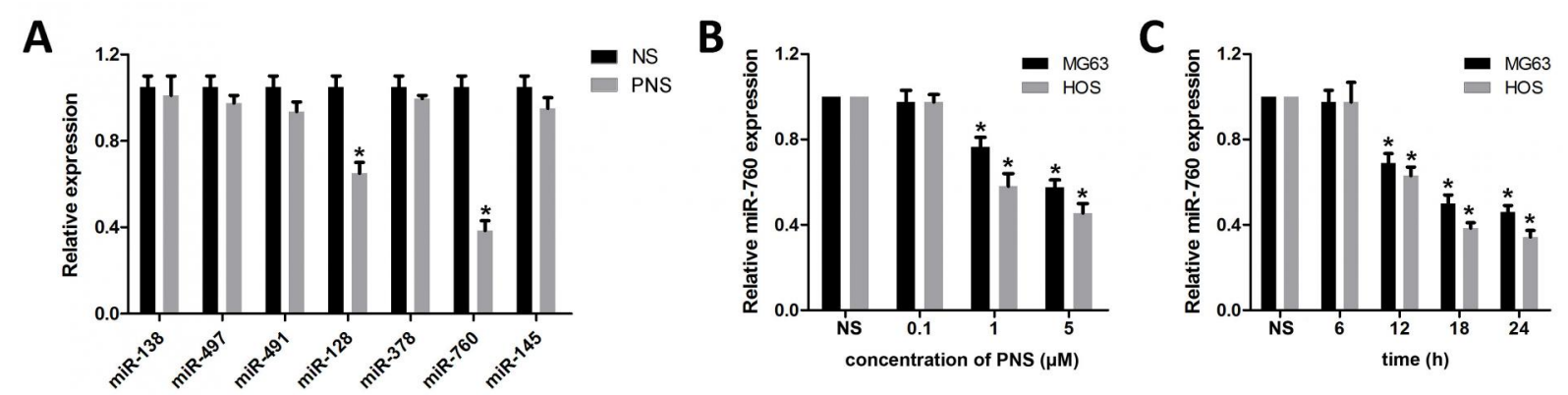


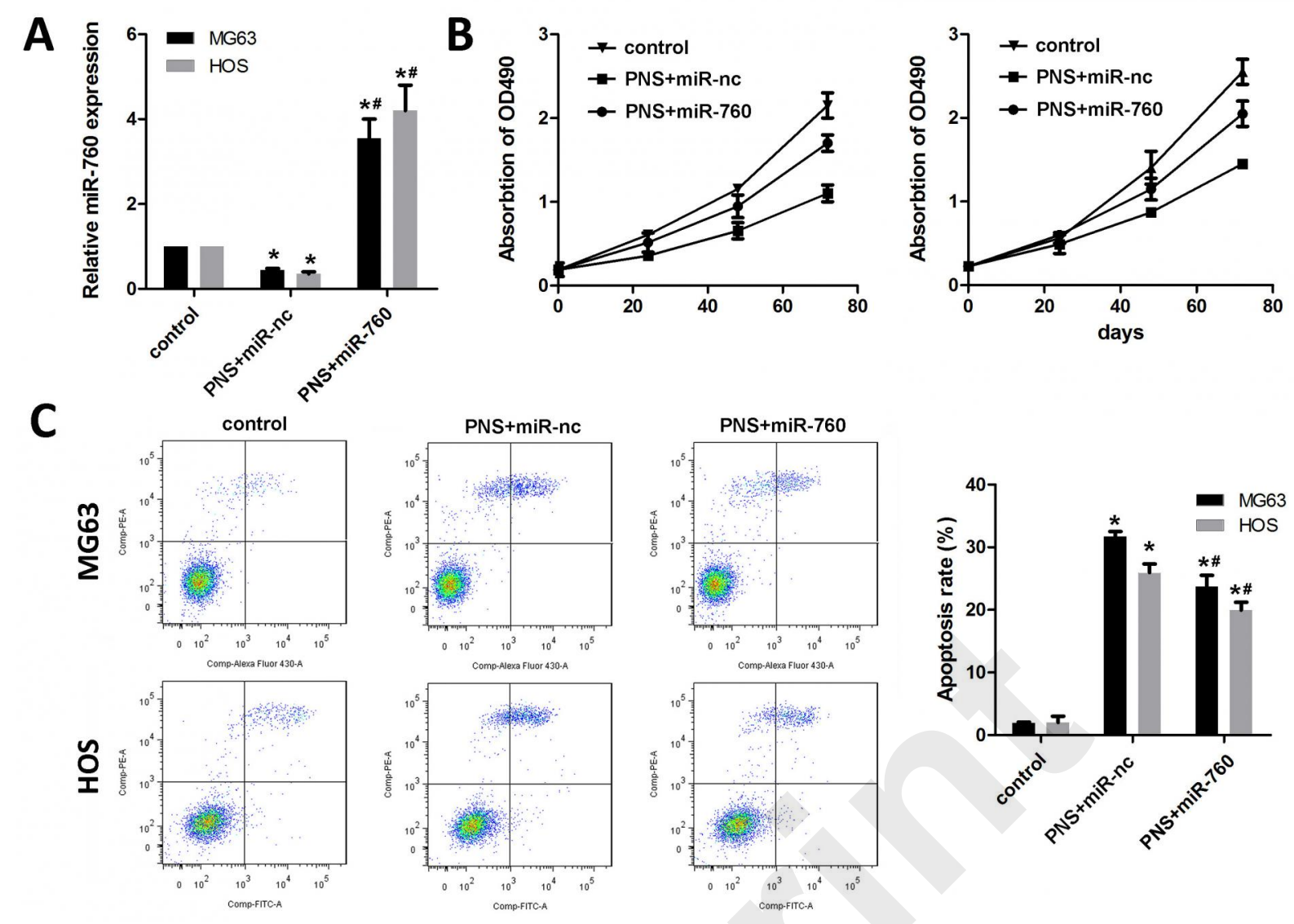


A

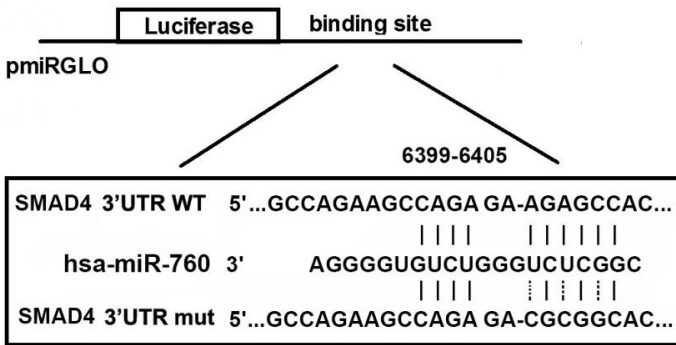

C

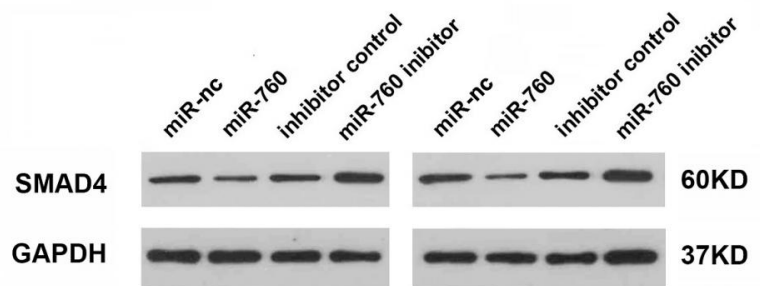

B

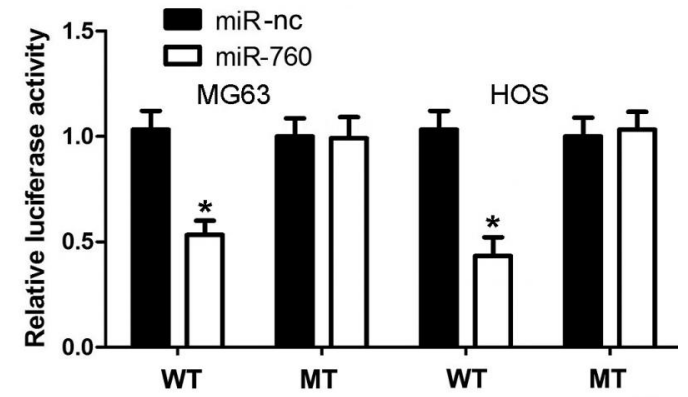

D

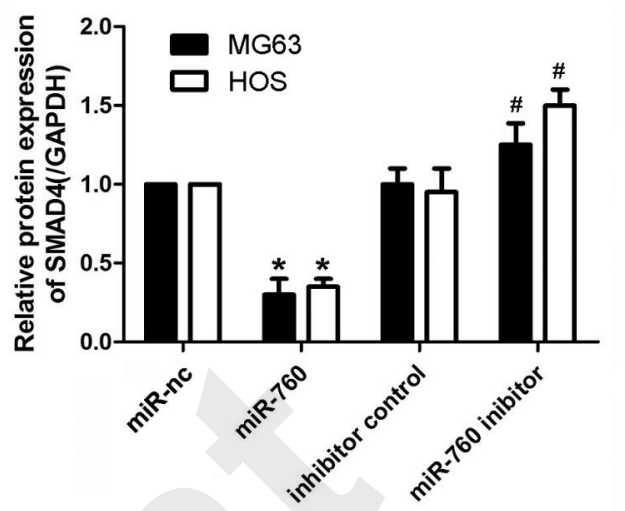




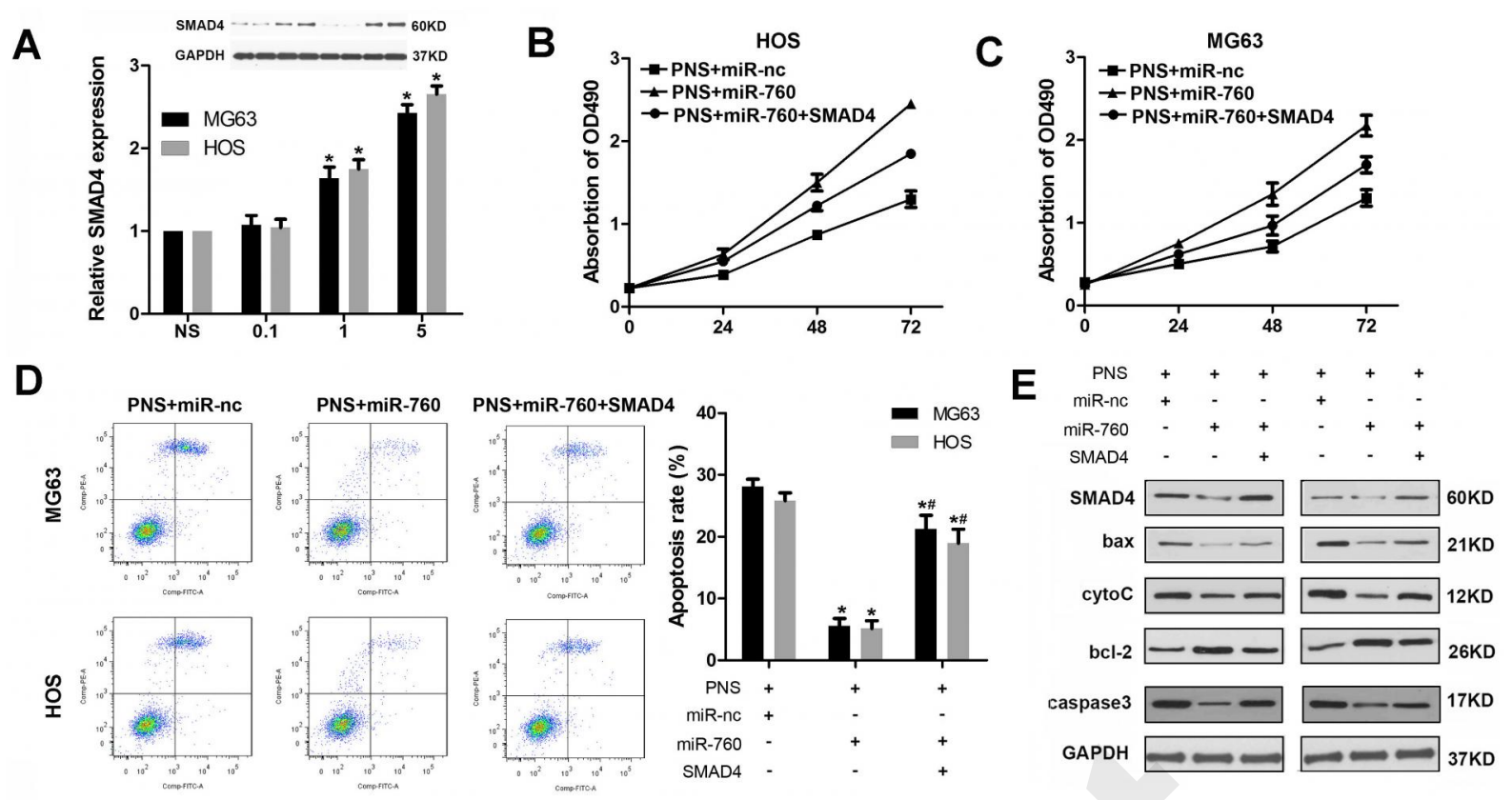


A

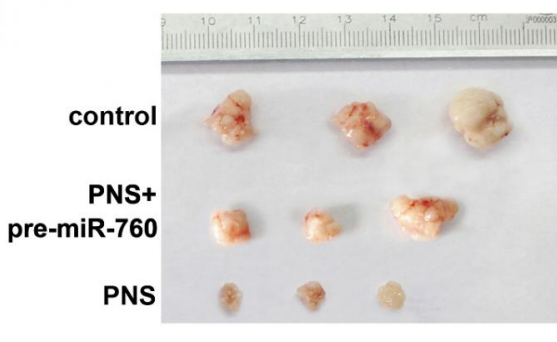

D

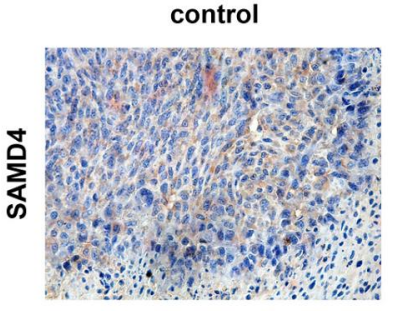

B

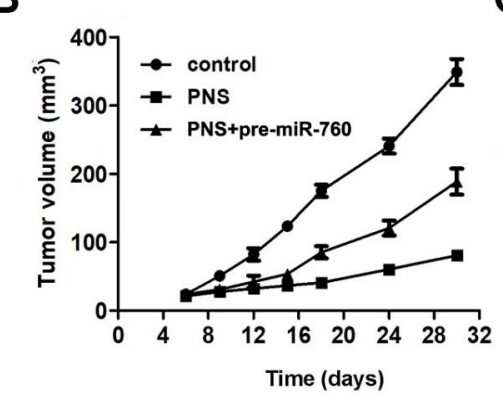

PNS

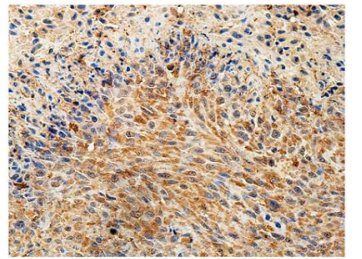

C

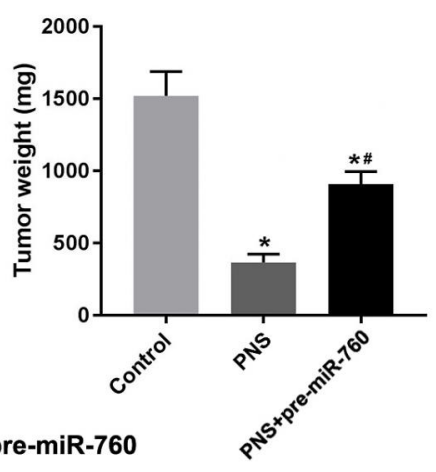

